ИЗВЕСТИЯ АКАДЕМИИ НАУК ЭСТОНСКОИ ССР. ФИЗИКА * МАТЕМАТИКА

PROCEEDINGS OF THE ACADEMY OF SCIENCES OF THE ESTONIAN SSR.

PHYSICS * MATHEMATICS

$1988,37,4$

УдК 514.75

U. LUMISTE and M. VÄLJAS

\title{
TOTALLY QUASIUMBILICAL SUBMANIFOLDS WITH NONFLAT NORMAL CONNECTION
}

C. LUMISTE, M. VALJAS. MITTETASASE NORMAALSEOSTUSEGA TAIELIKULT KVAASIOMBILISED ALAMMUUTKONNAD

Ю. ЛУМИСТЕ, М. ВЯЛЬЯС. ВПОЛНЕ КВАЗИОМБИЛИЧЕСКИЕ ПОДМНОГООБРАЗИЯ С НЕПЛОСКОП НОРМАЛЬНОП СВЯЗНОСТЬЮ

\section{(Presented by H. Keres)}

B.-Y. Chen and T. H. Teng have stated (see [ $\left.{ }^{1}\right]$, Theorem 1) that a totally quasiumbilical submanifold in a conformally flat Riemannian space has always flat normal connection. This statement is used in $\left[{ }^{2}\right]$, where Theorem 4 (the main theorem) says that an $n$-dimensional submanifold in an $(n+m)$-dimensional conformally flat space $(m<n-2)$ is totally quasiumbilical if and only if it is a conformally flat submanifold with flat normal connection. Remark that Errata corrige of $\left[{ }^{2}\right]$ doesn't concern this main theorem.

In this note we show that the Theorem 1 in [ $\left.{ }^{1}\right]$ and consequently the corresponding part (given above in the spaced type) of the Theorem 4 in $\left.{ }^{2}\right]$ are not true. We detect the mistake made in $\left.{ }^{1}\right]$ and give a counterexample (see $\$ 1$ and the theorem in $\$ 4$.)

$\$ 1$. In the «proof» of Theorem 1 in [ $\left.{ }^{1}\right]$ a general formula concerning the Weyl conformal curvature tensor $C_{j j, k l}$ of a submanifold in a conformally flat space is used. It is known (see $\left[^{3}\right]$ p. 150) that in this case $C_{i j, k l}=\sum_{\alpha} C_{i j, k l}^{\alpha}$ and thus $\sum_{i, j, k, l} h_{i k}^{\beta} h_{j l}^{\beta} C_{i j, k l}=\sum_{\alpha} \sum_{i, j, k, l} h_{i k}^{\beta} h_{j l}^{\beta} C_{i j, k l}^{\alpha}$. Straightforward computation gives that by $\alpha \neq \beta$ we have

$$
\begin{aligned}
(n-1) & (n-2) \sum_{i, j, k, l} h_{i k}^{\beta} h_{j l}^{\beta} C_{i j, k l}^{\alpha}=(n-1)(n-2)\left[\left(\left(H^{\beta} H^{\alpha}\right)_{[1]}\right)^{2}-\right. \\
& \left.-\left(H^{\beta} H^{\alpha}\right)_{[2]}\right]+\left[H_{[2]}^{\beta}-\left(H_{[1]}^{\beta}\right)^{2}\right]\left[H_{[2]}^{\alpha}-\left(H_{[1]}^{\alpha}\right)^{2}\right]+ \\
& +2(n-1)\left[H_{[1]}^{\alpha}\left(H^{\beta} H^{\beta} H^{\alpha}\right)_{[1]}+H_{[1]}^{\beta}\left(H^{\beta} H^{\alpha} H^{\alpha}\right)_{[1]}-\right. \\
& -H_{[1]}^{\beta} H_{[1]}^{\alpha}\left(H^{\beta} H^{\alpha}\right)_{[1]}-\left(H^{\beta} H^{\alpha} H^{\alpha} H^{\beta}\right)_{[1]},
\end{aligned}
$$

where $H_{[p]}=\operatorname{trace} H^{p}$ for a matrix $H$ and $H^{\alpha}=\left\|h_{i j}^{\alpha}\right\|$. In [ [1] the last term is replaced by $\left(H^{\beta} H^{\alpha}\right)_{[2]}$ and added to corresponding term in the first square bracket. Therefore in $\left[{ }^{1}\right]$ the formulas (3.6) and (3.9) are not valid and the proof of the theorem 1 is not correct. Instead of the decision inequality we have an identity.

$\S 2$. In fact this theorem is not true. In the following we give an example of a totally quasiumbilical submanifold $V^{n}$ in $E^{n+2}$ with nonflat normal connection. Note that a normal vector $\vec{\xi}$ is called quasiumbilical if $\sum_{\alpha} \xi^{\alpha} h_{i j}^{\alpha}$ has an $(n-1)$-fold eigenvalue and a $V^{n}$ in $E^{n+m}$ is called 
totally quasiumbilical if it has $m$ mutually orthogonal quasiumbilical unit normal vector fields (see $\left[{ }^{3}\right]$, Chap. 5).

$\S 3$. Let us start with two lemmas.

$\mathrm{L}$ e $\mathrm{mma}$ 1. Let $V^{2}$ be a surface with nonflat normal connection in $E^{4}$ and $V^{n}=V^{2} \times E^{n-2}$ its product by $E^{n-2}$ in $E^{n+2}, n>2$. Then the unit normal vector of $V^{n}$ in the point $X \in V^{n}$, orthogonal to the tangent line from $X$ to the normal curvature ellipse of the component $V^{2}$ in the plane of this ellipse, form a quasiumbilical unit vector field on $V^{n}$ (real if every $X \in V^{n}$ is not inside this ellipse).

Proof. We take the orthonormal frame vectors in the point $X \in V^{n}$ so that $\vec{e}_{1}$ and $\vec{e}_{2}$ are tangent to the $V^{2}$ and $\vec{e}_{3}, \ldots, \vec{e}_{n}$ lie in $E^{n-2}$. Then (see [4]) $d \overrightarrow{e_{u}}=\omega_{u}^{v} \overrightarrow{e_{v}} ; u, v=3, \ldots, n$ and in $d \overrightarrow{e_{1}}=\omega_{1}^{2} \vec{e}_{2}+\omega_{1}^{\alpha} \vec{e}_{\alpha}, d \overrightarrow{e_{2}}=$ $=-\omega_{1}^{2} \overrightarrow{e_{1}}+\omega_{2}^{\alpha} \vec{e}_{\alpha} ; \quad \alpha=n+1, n+2$, we have

$$
\omega_{1}^{\alpha}=h_{11}^{\alpha} \omega^{1}+h_{12}^{\alpha} \omega^{2}, \quad \omega_{2}^{\alpha}=h_{12}^{\alpha} \omega^{1}+h_{22}^{\alpha} \omega^{2} .
$$

The normal curvature vector in the direction of the unit tangent vector $\vec{t}=t \overrightarrow{e_{i}} ; \quad i=1, \ldots, n$, is

$$
\vec{h}_{i j} t i t j=\lambda\left(\vec{h}_{11} \cos ^{2} \varphi+2 \vec{h}_{12} \cos \varphi \sin \varphi+\vec{h}_{22} \cos ^{2} \varphi\right),
$$

where $\vec{h}_{i j}=h_{i j}^{n+1} \vec{e}_{n+1}+h_{i j}^{n+1} \vec{e}_{n+2}, \vec{h}_{1 u}=\vec{h}_{2 v}=\vec{h}_{u v}=0, t^{1}=\lambda \cos \varphi, t^{2}=\lambda \sin \varphi$, $0 \leqslant \lambda \leqslant 1, \lambda^{2}+\left(t^{3}\right)^{2}+\ldots+\left(t^{n}\right)^{2}=1$. Thus the normal curvature indicatrix of the submanifold $V^{n}$ in the point $X \in V^{n}$ (see [5], p. 109) is the convex hull of this point $X$ and of the normal curvature ellipse, which corresponds to $\lambda=1$ and whose point has the radius vector

$$
\frac{1}{2}\left(\vec{h}_{11}+\vec{h}_{22}\right)+\frac{1}{2}\left(\vec{h}_{11}-\vec{h}_{22}\right) \cos 2 \varphi+\vec{h}_{12} \sin 2 \varphi
$$

from the origin $X$ (see $\left[{ }^{6}\right]$, p. 253). In the frame with origin in the endpoint of mean curvature vector and with basic vectors $\frac{1}{2}\left(\vec{h}_{11}-\vec{h}_{22}\right)$ and $\vec{h}_{12}$ (if normal curvature is nonflat, they are noncollinear) this ellipse has parametric equations

$$
x=\cos 2 \varphi, \quad y=\sin 2 \varphi
$$

and thus these basic vectors have conjugated directions with respect to the ellipse. If the point $X \in V^{n}$ is not in the interior of this ellipse, then the frame vector $\vec{e}_{1}$ can be chosen so that the corresponding normal curvature vector $\vec{h}_{11}$ goes from $X$ in the tangent direction to the ellipse and ends in the point of tangency. The normal curvature vector $\vec{h}_{22}$, corresponding to $\vec{e}_{2}$, goes from $X$ to the diametrial point of the ellipse, so that $\frac{1}{2}\left(\vec{h}_{11}-\vec{h}_{22}\right)$ has diametrial direction. Thus $\vec{h}_{11}$ and $\vec{h}_{12}$ are collinear. Taking $\vec{e}_{3}$ in their common direction we have $h_{11}^{4}=h_{12}^{4}=0$ and so

$$
\operatorname{diag}\left\|h_{i j}^{4}\right\|=\left(0, h_{22}^{4}, 0, \ldots, 0\right) \text {. }
$$


The frame vector $\vec{e}_{4}$ forms now a quasiumbilical unit vector field. The lemma is proved.

Lemma 2. There exist locally euclidean surfaces $V^{2}$ with nonflat normal connection in $E^{4}$.

Proof. If $V^{2}$ in $E^{4}$ has Gaussian curvature $K=0$ and its normal connection is nonflat, then $X \in V^{2}$ lives on the orthooptical circle of the normal curvature ellipse (see $\left.{ }^{6}\right]$, p. 257). We can take the orthonormal frame as in the proof of Lemma 1 and have then $h_{11}^{4}=h_{12}^{4}=0, h_{22}^{4}=\sigma \neq 0$. The well-known formula $K=\sum_{\alpha}\left[h_{11}^{\alpha} h_{22}^{\alpha}-\left(h_{12}^{\alpha}\right)^{2}\right]$ gives now that $h_{11}^{3} h_{22}^{3}=\left(h_{12}^{3}\right)^{2}$, and there exist $\varrho$ and $\Theta$ so that $0<\Theta<\pi$ and

$$
h_{11}^{3}=\varrho \cos ^{2} \Theta, \quad h_{12}^{3}=\varrho \sin \Theta \cos \Theta, \quad h_{22}^{3}=\varrho \sin ^{2} \Theta .
$$

For the surface $V^{2}$ we have now the next differential system:

$$
\begin{gathered}
\omega^{3}=\omega^{4}=0, \\
\omega_{1}^{3}=\varrho \cos \Theta \cdot \omega_{\Theta}^{1}, \quad \omega_{2}^{3}=\varrho \sin \Theta \cdot \omega_{\Theta}^{1}, \\
\omega_{1}^{4}=0, \quad \omega_{2}^{4}=\sigma \omega^{2},
\end{gathered}
$$

where

$$
\omega_{\Theta}^{1}=\omega^{1} \cos \Theta+\omega^{2} \sin \Theta .
$$

By the exterior differentiation we get the covariant system

$\left[d_{\varrho} \cdot \cos \Theta-\varrho \sin \Theta \cdot\left(d \Theta+\omega_{1}^{2}\right)\right] \wedge \omega_{\Theta}^{1} \mid+\varrho \cos \Theta \cdot\left(d \Theta+\omega_{1}^{2}\right) \wedge \omega_{\Theta}^{2}=0$,

$$
\begin{gathered}
{\left[d_{\varrho} \cdot \sin \Theta+\varrho \cos \Theta \cdot\left(d \Theta+\omega_{1}^{2}\right)+\sigma \sin \Theta \cdot \omega_{3}^{4}\right] \wedge \omega_{\Theta}^{1}+} \\
+\left[\varrho \sin \Theta \cdot\left(d \Theta+\omega_{1}^{2}\right)+\sigma \cos \Theta \cdot \omega_{3}^{4}\right] \wedge \omega_{\Theta}^{2}=0,
\end{gathered}
$$

$\left[\sigma \sin \Theta \cdot \omega_{1}^{2}-\varrho \cos \Theta \cdot \omega_{3}^{4}\right] \wedge \omega_{\Theta}^{1}+\sigma \cos \Theta \cdot \omega_{1}^{2} \wedge \omega_{\Theta}^{2}=0$,

$\left[d \sigma \sin \Theta-\sigma \cos \Theta \cdot \omega_{1}^{2}+\varrho \sin \Theta \cdot \omega_{3}^{4}\right] \wedge \omega_{\Theta}^{1}+$

$$
+\left[d \sigma \cdot \cos \Theta+\sigma \sin \Theta \cdot \omega_{1}^{2}\right] \wedge \omega_{\Theta}^{2}=0,
$$

where

$$
\omega_{\Theta}^{2}=-\omega^{1} \sin \Theta+\omega^{2} \cos \Theta .
$$

Here we have 5 basic secondary forms: $d \varrho, d \Theta+\omega_{1}^{2}, \omega_{3}^{4}, \omega_{1}^{2}$ and $d \sigma$. The polar matrix has rank $s_{1}=4$ and $s_{2}=1$. Using Cartan lemma with basic primary forms $\omega_{\Theta}^{1}$ and $\omega_{\Theta}^{2}$ we get from the first pair of covariants 4 expressions with 6 new coefficients. Left sides of these expressions contain only 3 basic secondary forms and therefore 2 linear dependencies arise for those 6 coefficients. The next two covariants contain each a new basic secondary form and give 2 new coefficients. The general 2-dimensional integral element depends on $s_{1}+2 s_{2}=6$ arbitrary parameters. By Cartan theory $\left[{ }^{4}\right]$ the considered differential system is compatible and the lemma is proved.

Remark 1 . The normal curvature 2 -form of the $V^{2}$ in Lemma 2 is

$$
\Omega_{3}^{4}=-\varrho \sigma \cos \Theta \sin \Theta \omega^{1} \wedge \omega^{2} .
$$

Remark 2. The differential system in the proof of Lemma 2 gives the general locally euclidean $V^{2}$ with nonflat normal connection in $E^{4}$. If we add to its equations the next two new equations:

$$
d_{\varrho}=0, \quad d \Theta+\omega_{1}^{2}=0
$$


we get a differential system for a special case of those $V^{2}$ in $E^{4}$ with covariant system

$$
\begin{gathered}
\omega_{3}^{4} \wedge \omega^{4}=0 \\
\varrho \cos ^{2} \Theta \cdot \omega_{3}^{4} \wedge \omega^{1}+\sigma d \Theta \wedge \omega^{2}=0 \\
\sigma d \Theta \omega^{1}+d \sigma \wedge \omega^{2}+\varrho \sin \Theta \cos \Theta \cdot \omega_{3}^{4} \wedge \omega^{1}=0 .
\end{gathered}
$$

This system has 3 basic secondary forms and its polar matrix has rank $s_{1}=3$. The compatibility of the differential system follows immediately from the corresponding Cartan theorem $\left(\left[{ }^{4}\right]\right.$, p. 94).

$\S 4$. Now we give a counter-example to Theorem 1 in $\left[{ }^{1}\right]$, which shows that this theorem is false.

Theorem. There exist totally quasiumbilical submanifolds $V^{n}$ with nonflat normal connection in $E^{n+2}$.

P r o of. Let $V^{2}$ be a locally euclidean surface with nonflat normal connection in $E^{4}$ (see Lemma 2, Remarks 1 and 2). Its point $X \in V^{2}$ lies on the orthooptical circle of the normal curvature ellipse. Thus tangent lines from $X$ to the ellipse are orthogonal. Due to the Lemma 1 unit vectors in directions of these two lines form two orthogonal quasiumbilical normal vector fields on the product $V^{n}=V^{2} \times E^{n-2}$ in $E^{n+2}$. Therefore $V^{n}$ is totally quasiumbilical and has nonflat normal connection. The theorem is proved.

\section{REFERENCES}

1. Chen, B.-Y., Teng, T. H. // Soochow J. Math. and Natur. Sci., 1975, 1, № 1, 9-16.

2. Chen, B.-Y., Verstraelen, L. // Boll. Unione math. ital., 1977, A14, № 1, 49-57; Errata corrige: Boll. Unione math. ital., 1977, A14, № 3, 634.

3. Chen, B.-Y. Geometry of Submanifolds. New York, M. Dekker, 1973.

4. Картан Э. Внешние дифференциальные системы и их геометрические приложения. М., МГУ, 1962.

5. Схоутен И. А., Стройк Д. Дж. Введение в новые методы дифференциальной геометрии. М., ИЛ. 1948, 2.

6. Картан Э. Риманова геометрия в ортогональном репере. М., МГУ, 1960.

Tartu State University

Received

Feb. 23, 1988 